\title{
CROP PHENOLOGY CLASSIFICATION USING A REPRESENTATION LEARNING NETWORK FROM SENTINEL-1 SAR DATA
}

\author{
Subhadip Dey ${ }^{l}$, Dipankar Mandal ${ }^{1}$, Vineet Kumar ${ }^{l}$, Biplab Banerjee ${ }^{1}$, \\ J. M. Lopez-Sanchez ${ }^{2}$,Heather McNairn ${ }^{3}$, Avik Bhattacharya ${ }^{1}$ \\ ${ }^{1}$ Centre of Studies in Resources Engineering, \\ Indian Institute of Technology Bombay, Mumbai, India \\ ${ }^{3}$ University of Alicante, Alicante, Spain \\ ${ }^{2}$ Agriculture and Agri-Food Canada, Ottawa, Canada
}

\begin{abstract}
This work deals with the classification of wheat phenology by regressing the synthetic aperture radar (SAR) backscatter coefficients $(\mathrm{VV}, \mathrm{VH})$ to vegetation water content $(\mathrm{VWC})$ and plant area index (PAI) through a representation learning network. The representation network architecture consists of a pair (VV, VH) of two regression layers (VWC, PAI) which finally converge to a classification (crop phenology) layer. The study was conducted with the Sentinel-1 C-band SAR data acquired during the SMAPVEX16 campaign in Manitoba, Canada. Using this framework, the wheat phenology was classified to an accuracy of $86.67 \%$. However, in comparison, the classification accuracy reduced by $\sim 20 \%$ while using only the backscatter coefficients of $(\mathrm{VV}, \mathrm{VH})$ polarization channels. The results obtained from this study justifies the potential of using a representation learning scheme for crop phenology classification with SAR data.
\end{abstract}

Index Terms - Wheat; Phenology Classification; Sentinel1; Neural network; SMAPVEX16

\section{INTRODUCTION}

Estimation of crop phenology is important for various groundlevel measurements: application of fertilizer, pesticide, and fungicide, tillage or soil preparation and time of harvest. Different types of fertilizers are required for better vegetative growth at different phenological stages of the crop. Besides this, the amount of water required in the field also varies throughout the crop growth stages.

There are several ground-based models to estimate wheat phenology which requires a large number of meteorological parameters like, precipitation [1], solar angle, solar radiation, temperature, humidity, soil parameter, soil texture and structure, soil moisture etc [2]. Accumulation and assimilation of

The authors would like to thank the SMAPVEX16-MB team for providing in-situ measurements and ESA for providing Sentinel-1 through Copernicus Open Access Hub. these parameters for large areas are non-trivial and merely feasible. Besides, the quality of data also deteriorates as the number of observations is increased. On the contrary, remote sensing techniques have been used successfully to estimate biophysical parameters and crop phenology information. In this context, optical remote sensing data have been used aptly in several studies with promising accuracy [3]. However, the main drawback of using optical remote sensing data lies in the fact that it gets affected by clouds, mist or even rainfall.

On the other hand, microwave sensors, with longer transmitted wavelength are usually unaffected by atmospheric conditions. The backscatter signal received by these sensors is highly influenced by surface roughness and dielectric properties of the soil and crop geometry. Hence, there is good potential for vegetation information extraction using radar remote sensing data. In response, an attempt has been made in [4] to show the potential of the dual-pol X-band SAR data (TerraSAR-X) for tracking rice phenology.

Improved estimation of rice growth stages was reported in [5] with a meta model-based approach with intense in-situ measurements of plant physiological descriptors. However, it is tedious to take into account discrete element-wise measurements like stalk height and diameter, leaf length and width, leaf count and tiller count from each field. Moreover, the resolution cell gets coarser from X-band to C-band data. Therefore, within a SAR resolution cell, this representation would be difficult to achieve. Alternatively, crop biophysical parameters e.g. Plant Area Index (PAI) and Vegetation Water Content (VWC) could provide better information of the canopy as a whole, instead of element wise measurement.

In a recent study, a good correlation between crop biophysical parameters (LAI/PAI and plant height) and Quadpol C-band SAR polarimetric observables was reported for different growth stages of crop [6]. It was suitably demonstrated that multi-temporal C-band polarimetric SAR parameters could be used for tracking phenological development stages. The rapid expansion of dual-pol SAR constellation satellites e.g. Sentinel-1, SAOCOM, and, the forthcoming 
NISAR, would facilitate phenology tracking due to rapid temporal revisit and wide swath coverage. However, the estimation of phenology is a challenge due to the lesser information content in dual-pol Sentinel-1 data compared to the data acquired by quad-pol sensors. Thus, a logical approach would be to estimate phenology using certain derived parameters from dual-pol data. Hence, in this study, we have proposed a representation learning technique to classify wheat phenology using dual-pol backscatter coefficients from Sentinel-1 sensor via the estimation of VWC and PAI.

\section{METHODOLOGY}

In the context of this work, a combination of regression and classification layers in a representational learning architecture is developed for the classification of wheat phenology from the backscatter coefficients of Sentinel-1 dual-pol (VV and VH) SAR data. The methodology is discussed in the following sections:

\subsection{Study area and in-situ measurements}

The in-situ measurements were carried out during June to July 2016 as a part of SMAPVEX16-MB validation campaign over the Red River watershed of southern Manitoba, Canada $\left(49^{\circ} 40^{\prime} 32^{\prime \prime} N, 97^{\circ} 59^{\prime} 57^{\prime \prime} W\right)$ as shown in Fig. 1. Wheat is one of the main cultivated crops in this area. Field sizes range from 20-30 to 50-60 hectares. The total cultivated area of spring wheat and winter wheat is $25.53 \%$.
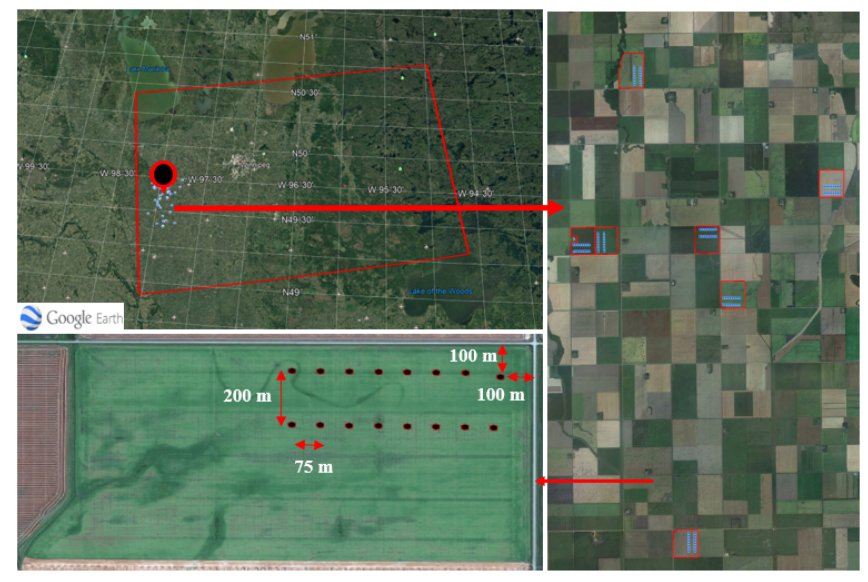

Fig. 1: Study area over the Red River watershed in Manitoba (Canada). Sampling locations during the SMAPVEX16-MB campaign in each field are shown in black points.

Depending on the soil textural condition and annual crop production, 13 wheat fields were selected for sampling. A total of 16 sampling points were allocated in two parallel transects in each field. The sampling points of the first row are $100 \mathrm{~m}$ away from the road and the second row is $200 \mathrm{~m}$ away from the previous one. Each sampling point in a particular row is $75 \mathrm{~m}$ away from each other and the distance between the last sampling point and the edge of the field is $100 \mathrm{~m}$ as illustrated in Fig. 1. A set of three sampling points from each field was conducted once per week.

The wheat biomass was collected by destructive sampling procedure. The above ground crop canopy portion is cut from a square area of $0.5 \mathrm{~m} \times 0.5 \mathrm{~m}$. The weight of the wet biomass was measured immediately as the vegetation moisture condition starts to deteriorate right after the cutting of the crop canopy. Subsequently, the dry biomass weight was also measured in the laboratory along with the Vegetation Water Content (VWC kgm ${ }^{-2}$ ) The Plant area index (PAI) was measured using the hemispherical lens. Seven photos of the top of the canopy of wheat fields along each of the two transects were measured and processed by CAN-EYE to generate the PAI [7].

\subsection{SAR data processing}

The C-band Sentinel-1 interferometric wide (IW) swath level1 GRD product is used in this study. Three Sentinel-1 images: Jun-Jul, 2016, acquired during the SMAPVEX16-MB campaign are utilized in the present study. The intensity image is calibrated to evaluate the backscatter coefficient. The images are terrain corrected and speckle filtered with a $3 \times 3$ boxcar filter. An image subset of the study area is used for analysis in this work.

\subsection{Dataset preparation}

The VV and VH backscatter coefficients are extracted from the geocoded image along with the in-situ biomass and PAI measurements. Any missing data from the image or any non-identified field data are removed from the sample. A total of 76 sample points are used for VWC, PAI, VH, VV and crop stage for the phenology classification of the wheat crop. Among these, 50 sample points are randomly chosen to regress the $\mathrm{VV}$ and $\mathrm{VH}$ backscatter coefficients to VWC and PAI. $80 \%$ of the data points are used for training while the rest $20 \%$ are used for validation. In the classification step, 60 data points are used for training while the rest is used for testing.

\subsection{Representation learning architecture}

The Representation Learning Architecture (RLA), proposed in this work is an interconnected chain of linear regression models. In general, a RLA can be expressed as,

$$
\begin{gathered}
X=\{x, y, z\}^{N} \\
f: x \rightarrow y \rightarrow z \\
f=\theta(w, b)
\end{gathered}
$$

where, $x$ is the input (VV, VH) variable, $y$ is the target (VWC, $\mathrm{PAI})$ variable, $z$ is the target crop phenology classes, $w$ is the 


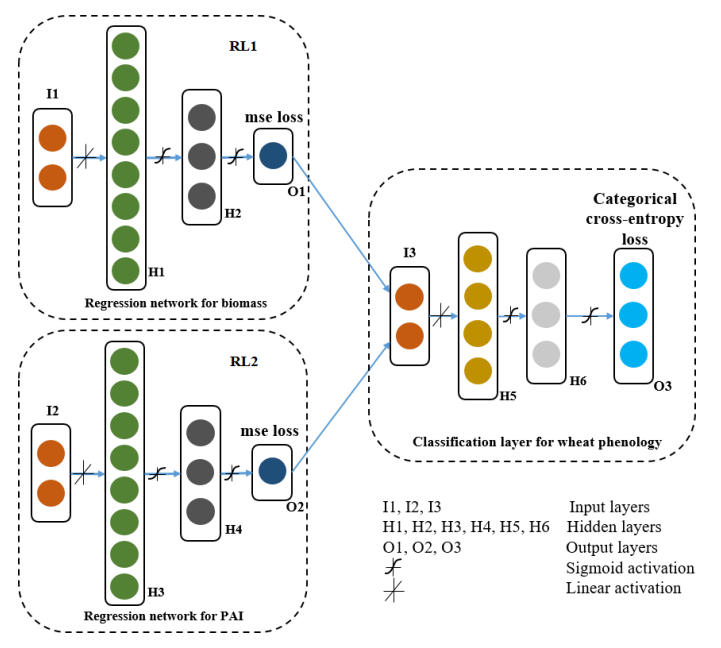

Fig. 2: Illustration of representation network architecture.

weight assigned to different input variables and $b$ is the bias. A function $f$ is used to learn $w$ and $b$.

The proposed architecture is similar to a backpropagation multi-layer perceptron [8]. A simple RLA is a combination of one or more regression layers (RL) and a classification layer (CL). The RL consists of an input layer, an output layer, and one or more hidden layers. The aim of this network is to minimize the cost function by updating the weights in between the connected nodes. In this work, the RL has an input layer $\left(R^{12}\right)$, two hidden layers ( $R^{18}$ and $R^{13}$ respectively) and an output layer $\left(R^{11}\right)$. The Sigmoid function is used as an activation function in the hidden layers. The hidden layers are used to transform the data so that the correlation between the target and the transformed data are increased.

The CL consist one input layer $\left(R^{12}\right)$, two hidden $\left(R^{14}\right.$ and $R^{13}$ respectively) layers and one output $\left(R^{13}\right)$ layer. The outputs of the RL are used as inputs to the CL for wheat phenology classification. The Sigmoid activation function with categorical cross entropy loss is used in CL. The overall architecture of the proposed model is illustrated in Fig. 2 The $\mathrm{VV}$ and $\mathrm{VH}$ backscatter coefficients are used as input features in both RL1 and RL2 blocks. The VWC and PAI are used as target parameter in RL1 and RL2 layers respectively. The output of RL1 and RL2 layers are taken as input to the CL block for phenology classification.

\section{RESULTS AND DISCUSSION}

Three different growth stages (BBCH scale) of wheat are used in this work. BBCH 13-15 is considered as stage-1. In this stage, the development of 3,4 or 5 leaves of wheat crops take place. Based on the available information, the number of crops in BBCH 14 was more than that in $\mathrm{BBCH}-13$ and BBCH-15. Hence, the value of PAI tends to be high. At Stage-1, the distribution of PAI is left-skewed as shown in the box-plot of Fig. 3. However, the box-plot of VWC shows

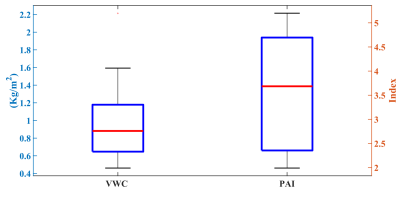

(a) Stage-1

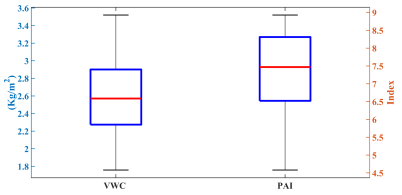

(b) Stage-2

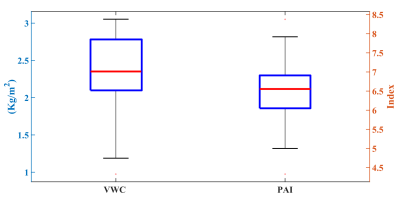

(c) Stage-3

Fig. 3: Growth stage wise distribution of VWC and PAI

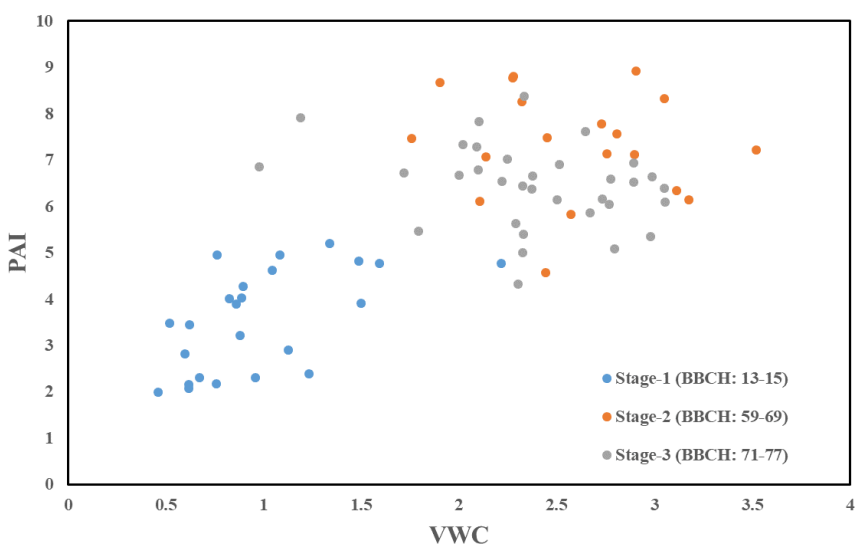

Fig. 4: Illustration of VWC vs. PAI dataset (Stage wise).

right skewness at stage-1. This may be due to the field condition in the study area. However, both VWC and PAI rapidly increases at this stage. A high correlation between VWC and PAI is apparent in Fig. 4 The growth stages corresponding to BBCH 59-69 are considered as Stage-2. This stage starts with the completion of the emergence of the head (59). Then, the initiation (60) and end (69) of floriation of wheat crops. Most of the fields were near to the half flower completion stage (65). Therefore, the box-plot in Fig. 3 shows the PAI to be left-skewed. For stage-3, BBCH 71-77, the development of the late milk stage is followed by the watery kernel. The early and medium milk stages take place during this stage. Due to the high population of medium and late milk stage of wheat crops, the box plot of PAI also shows a left-skewed result.

The LAI increases sharply during the initial stages of cereals. Besides, it could get reduced due to the variation in temperature. Hence, the median value of PAI increased rapidly from stage- 1 to stage- 2 . Whereas, it got reduced at stage3. Similarly, the VWC of cereals increases at initial stages. Thereafter, it tends to get saturated from the initiation of reproductive stages of cereals. Hence, there is a steep increase 
of VWC from stage-1 to stage-2, but it remains almost constant from stage-2 to stage-3.

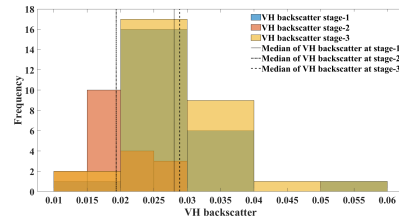

(a) $\mathrm{VH}$

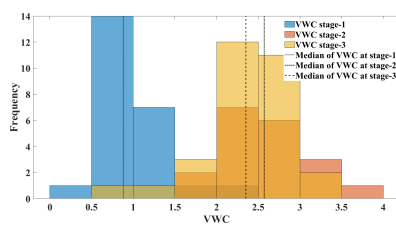

(c) VWC

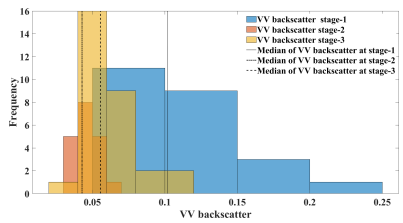

(b) VV

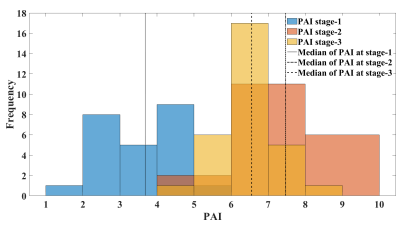

(d) PAI
Fig. 5: Histogram and median values of VH, VV, VWC and PAI.

Two RL segments are used in the network architecture as there is a change in the correlation between VWC and PAI throughout the wheat crop development period (Fig 2). The maximum obtained accuracy in the estimation of the phenology of wheat via VWC and PAI by the proposed model is $86.67 \%$. Whereas, it drastically falls down to $60 \%$ while classifying directly from the VV and VH backscatter coefficients. The enhancement of accuracy might be due to the more separation in between the three stages after regression to VWC and PAI from VV and VH (Fig 5).

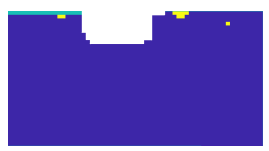

(a)

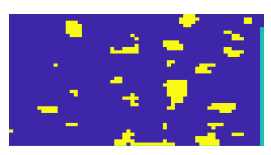

(d)

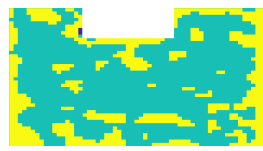

(b)

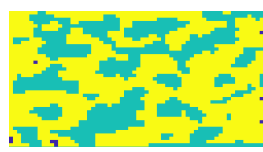

(e)

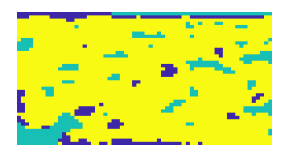

(c)

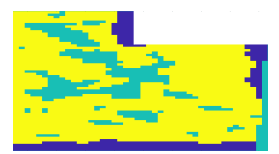

(f)
Fig. 6: Wheat phenology map over two representative field patches. Stage-1 (a, d), Stage-2 (b, e), Stage-3 (c, f).

The classified phenological stages of wheat are shown in Fig 6. Stage-1 wheat crop is very less mixed with other stages whereas, the estimated map of stage- 2 and stage- 3 are mixed with each other. This is due to the saturation of VWC and PAI after the initiation of reproductive stages of the wheat crop. The uncertainty in the classification accuracy is more during stage-2 due to the unavailability of adequate training and validation sample.

\section{CONCLUSIONS}

A novel neural network based representation architecture is proposed in this work for the classification of phenological growth stages of the wheat crop. The proposed architecture enhances the classification accuracy of the growth stages via the estimation of crop biophysical parameters such as vegetation water content (VWC) and plant area index (PAI). This is due to the better separation of the growth stages after regression to VWC and PAI from VH and VV backscatter coefficients. The proposed representation learning architecture can be suitably expanded to include input parameters from multiple remote sensing sensors. In addition to this, the flexibility of the architecture could be utilized to classify the phenological growth stages of other crops like pulse, forage etc.

\section{REFERENCES}

[1] Gerrit Hoogenboom, "Contribution of agrometeorology to the simulation of crop production and its applications," Agricultural and forest meteorology, vol. 103, no. 1-2, pp. 137-157, 2000.

[2] W Terink, P Droogers, J van Dam, G Simons, and M Voogt, "The added value of high-resolution above coarse-resolution remote sensing images in crop yield forecasting: a case study in the Egyptian Nile Delta," FutureWater report, vol. 116, 2012.

[3] Chunhua Liao, Jinfei Wang, Taifeng Dong, Jiali Shang, Jiangui Liu, and Yang Song, "Using spatio-temporal fusion of landsat-8 and modis data to derive phenology, biomass and yield estimates for corn and soybean," Science of The Total Environment, 2018.

[4] Juan M Lopez-Sanchez, Shane R Cloude, and J David BallesterBerman, "Rice phenology monitoring by means of SAR polarimetry at X-band," IEEE Transactions on Geoscience and Remote Sensing, vol. 50, no. 7, pp. 2695-2709, 2012.

[5] Onur Yuzugullu, Stefano Marelli, Esra Erten, Bruno Sudret, and Irena Hajnsek, "Determining rice growth stage with X-Band SAR: A metamodel based inversion," Remote Sensing, vol. 9, no. 5, pp. 460, 2017.

[6] Francis Canisius, Jiali Shang, Jiangui Liu, Xiaodong Huang, Baoluo Ma, Xianfeng Jiao, Xiaoyuan Geng, John M Kovacs, and Dan Walters, "Tracking crop phenological development using multi-temporal polarimetric Radarsat-2 data," Remote Sensing of Environment, vol. 210, pp. 508-518, 2018.

[7] Hassan AKM Bhuiyan, Heather McNairn, Jarrett Powers, Matthew Friesen, Anna Pacheco, Thomas J Jackson, Michael H Cosh, Andreas Colliander, Aaron Berg, Tracy Rowlandson, et al., "Assessing SMAP soil moisture scaling and retrieval in the Carman (Canada) study site," Vadose Zone Journal, vol. 17, no. $1,2018$.

[8] Simon S Haykin, Neural networks and learning machines, vol. 3, Pearson Upper Saddle River, 2009. 